Observations

of the

CALIFORNIA INSTITUTE OF TECHNOLOGY

RADIO OBSERVATORY

Owens Valley, California

1961

6. THE RADIO SPECTRUM OF SUPERNOVA REMNANTS

by

D. E. Harris 


\title{
THE RADIO SPECTRUM OF SUPERNOVA REMNANTS
}

\author{
by \\ D. E. Harris \\ California Institute of Technology \\ Radio Observatory \\ Owens Valley, California
}

\begin{abstract}
$960 \mathrm{Mc} / \mathrm{s}$ observations of thirteen galactic sources generally assumed to be supernova remnants, are coupled with previous observations at other frequencies to derive spectral indices. Although several values of spectral index are found in the neighborhood of zero, arguments are presented that free-free transitions are not the primary cause of the radio emission.

An interpretation of the relatively large range of spectral indices is suggested on the basis of an evolutionary sequence in which young, bright objects with relatively steep spectra gradually evolve into old, faint objects with flat or inverted spectra.

\section{Introduction}

The radio investigation of supernova remnants was initiated in 1949 when Bolton and Stanley (1949) identified Taurus A with the remains of the galactic supernova of 1054. Further radio work on supernovae has been hampered by the inability of radio techniques to resolve individual features of the distant galaxies where most known supernovae have occurred. Therefore, radio observations have concentrated on the three known galactic supernovae, together with an ever increasing number of extended galactic sources. The latter sources are non-thermal radio emitters with optical features which often display a general expansion. Table l lists all such objects with which we are familiar.

The present paper is based on part of the author's thesis and is a study of the first thirteen sources listed in Table 1 . These thirteen sources, hereafter assumed to be supernova remnants, were observed at $960 \mathrm{Mc} / \mathrm{s}$ with a $90^{\prime}$ parabolic antenna at the owens Valley Radio Observatory. The receiver and the observing procedures have been described elsewhere (Harris and Roberts 1960; hereafter referred to as "CTA").
\end{abstract}


Table 1 - Possible Supernova Remnants

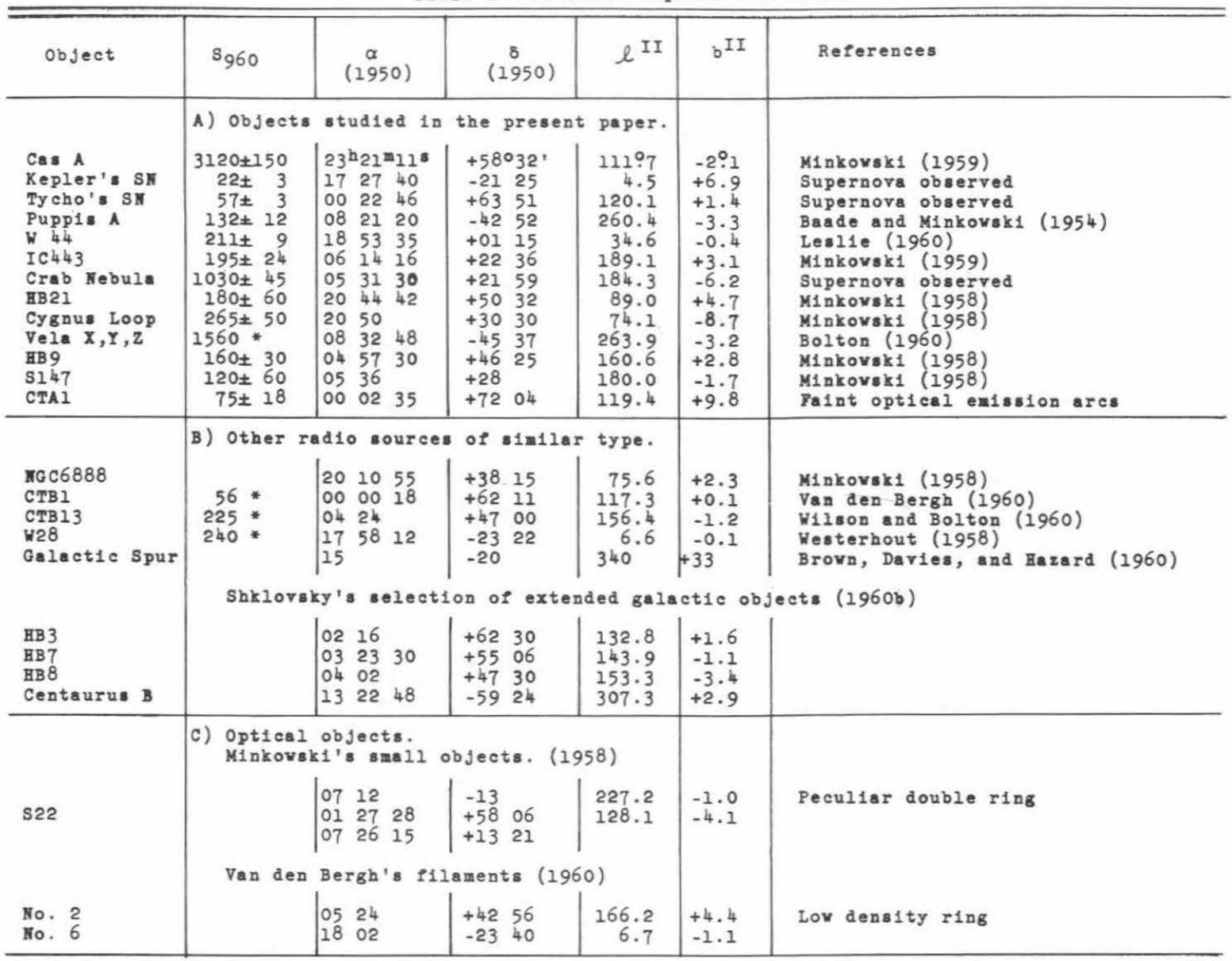

S960 $1 \mathrm{~s}$ the flux density at $960 \mathrm{Mc} / \mathrm{s}$ measured in units of $10^{-26} \mathrm{Wm}^{-2}(\mathrm{c} / \mathrm{s})^{-1}$.

* CTB plux density used (W1lson and Bolton 1960). 
The smaller supernova remnants have been studied previously by many workers and do not lend themselves to extensive investigation with the present equipment. Therefore, most of the observational effort has been spent on the larger objects.

The Cygnus Loop is one of the strongest sources of this type at $960 \mathrm{Mc} / \mathrm{s}$ and has been examined previously in more detail than most other similar remnants. Furthermore, it is significantly resolved with beamwidths such as ours (0!8). For these reasons, the Cygnus Loop is presented in this paper as a prototype of the larger supernova remnants.

In Section II, $960 \mathrm{Mc} / \mathrm{s}$ flux densities are used in conjunction with measurements of various observers at other frequencies to determine spectra relative to the standard source, M87. Since the derived spectra of many large supernova remnants are characteristic of HII regions, calculations based on optical observations are made to determine the extent of radio emission from free-free transitions. These calculations, given in Section III, indicate that most of the radio emission must be non-thermal.

Section IV presents evidence which suggests that the spectral index of any remnant varies with time. This hypothesis is based on empirical correlations found for the 13 objects studied in this paper. When the spectral indices of the various remnants are plotted against the appropriate value of linear diameter or radio surface brightness, it appears that an initially steep spectrum may flatten with age.

\section{Observations and Spectra}

\section{a) Intensity Measurements of the Cygnus Loop}

The present observations of the Cygnus Loop consist of about 30 drift curves of 40 minutes each taken at one-half degree intervals between $+27^{\circ} 30^{\prime}$ and $+33^{\circ} 30^{\prime}$. These intensity records, obtained between April and November 1959, were normalized by a drift curve taken each night at $\delta=+30000^{\prime}$. Figure 6 (Appendix III) shows the resulting Cygnus Loop contours of antenna temperature where a linear subtraction for the galactic background has been made. The galactic correction was performed on the average drift curves by noting the slope of the record of antenna temperature as the galaxy left the antenna beam before the source was encountered.

Following Mathewson, Large and Haslam (1961), hereafter referred to as "MLH", the Loop was divided into three parts: A, B, and C. (The boundaries are indicated by the dotted lines in Figure 6.) Each part was integrated with a planimeter, in order to derive a value of intensity for the individual sections as well as for the complete source. The intensity published earlier (CTA) was based on a preliminary contour diagram and the value given here supercedes that.

The maximum intensity occurred on the $\delta=+30^{\circ} 00^{\prime}$ records (Source A) and the average of 7 observations gives a value of $0.104 \pm 0.008$ (p.e.) for the peak intensity relative to M87. The flux density from the entire source is then found to be $0.88 \pm 0.17$ times that of M87 
$\left[(265 \pm 50) 10^{-26} \mathrm{Wm}^{-2}(\mathrm{c} / \mathrm{s})^{-1}\right.$ if $\left.\mathrm{S}_{M 87}=300 \cdot 10^{-26} \mathrm{wm}^{-2}(\mathrm{c} / \mathrm{s})^{-1}\right]$. The error of $\pm 20 \%$ consists of $12 \%$ from the integration process and $8 \%$ from the scatter in the observed peak intensity.

\section{b) Spectrum of the Cygnus Loop}

Observations made at other frequencies are presented in Table 2 . For the purpose of deriving a spectral index, published flux densities have been normalized to the standard source (M87) whenever possible. The secondary calibrators Cas A and 3C 295 were used to relate Westerhout's $400 \mathrm{Mc} / \mathrm{s}$ measurement and the MLH values, respectively, to M87. The adopted spectra are

$$
\begin{aligned}
& S_{M 87}=42,000 f^{-0.72} \\
& S_{\text {Cas A }}=757,000 \mathrm{f}^{-0.8} \\
& S_{295}=948 \mathrm{f}^{-0.5}
\end{aligned}
$$

Whitfield, 1957

CTA

where $S$ is in $10^{-26} \mathrm{Wm}^{-2}(\mathrm{c} / \mathrm{s})^{-1}$ and $f$ is in $\mathrm{Mc} / \mathrm{s}$. This normalizing procedure should not be viewed as a correction of published flux densities; rather it is a method of deriving the spectrum of the Cygnus Loop relative to that chosen for M87.

The adopted flux densities are plotted in Figure 1. The interpretation in terms of spectral index must rest on a subjective choice of which data to weight most heavily. MLH have suggested that Westerhout's flux density of the Cygnus Loop at $1390 \mathrm{Mc} / \mathrm{s}$ may be low if the weaker sections of the source were not seen. The same comment may be made of the $915 \mathrm{Mc} / \mathrm{s}$ value of Eaton and Kraus. Costain's measurement at $38 \mathrm{Mc} / \mathrm{s}$ employed a corner reflector with a $40^{\circ}$ beam to half power points in declination. As HB2l is only $20^{\circ}$ away, and the galaxy is even closer, his intensity measurement may suffer from confusion.

If the spectrum follows a simple power law as assumed in Figure 1 , the spectral index is $x_{C L}=-0.10 \pm 0.15$. The error quoted for this value was estimated by fitting lines of various slopes to the data of Figure 1, and is a probable error.

The relative spectral index of each part of the Cygnus Loop may be obtained by comparing the contour diagrams at 408 and $960 \mathrm{Mc} / \mathrm{s}$. Fortuitously, our $960 \mathrm{Mc} / \mathrm{s}$ beamwidth is almost identical to that of the $250^{\prime}$ telescope at $408 \mathrm{Mc} / \mathrm{s}$. Therefore, the MLH contour map was divided by the same boundaries as the $960 \mathrm{Mc} / \mathrm{s}$ map and the same integration procedure was used to evaluate each source. It was found that each of the three parts contribute essentially the same percentage to the total emission as it did at $960 \mathrm{Mc} / \mathrm{s}$. This conclusion is confirmed for Source C by the estimate of Baldwin and Leslie (1960) that 40 of the total $430 \cdot\left(10-26 \mathrm{Wm}^{-2}(\mathrm{c} / \mathrm{s})-1\right)$ at $178 \mathrm{Mc} / \mathrm{s}$ could be attributed to the eastern hump in their contours; 1 .e. Source $C$.

Table 3 lists the pertinent data. First it compares the $960 \mathrm{Mc} / \mathrm{s}$ positions with the $408 \mathrm{Mc} / \mathrm{s}$ positions. Then the results of the integrations are given and spectral indices of $\mathrm{A}, \mathrm{B}$, and $\mathrm{C}$ are computed relative to the spectral index of the entire Loop. 
Table 2 - Flux Densities of Supernova Remnants

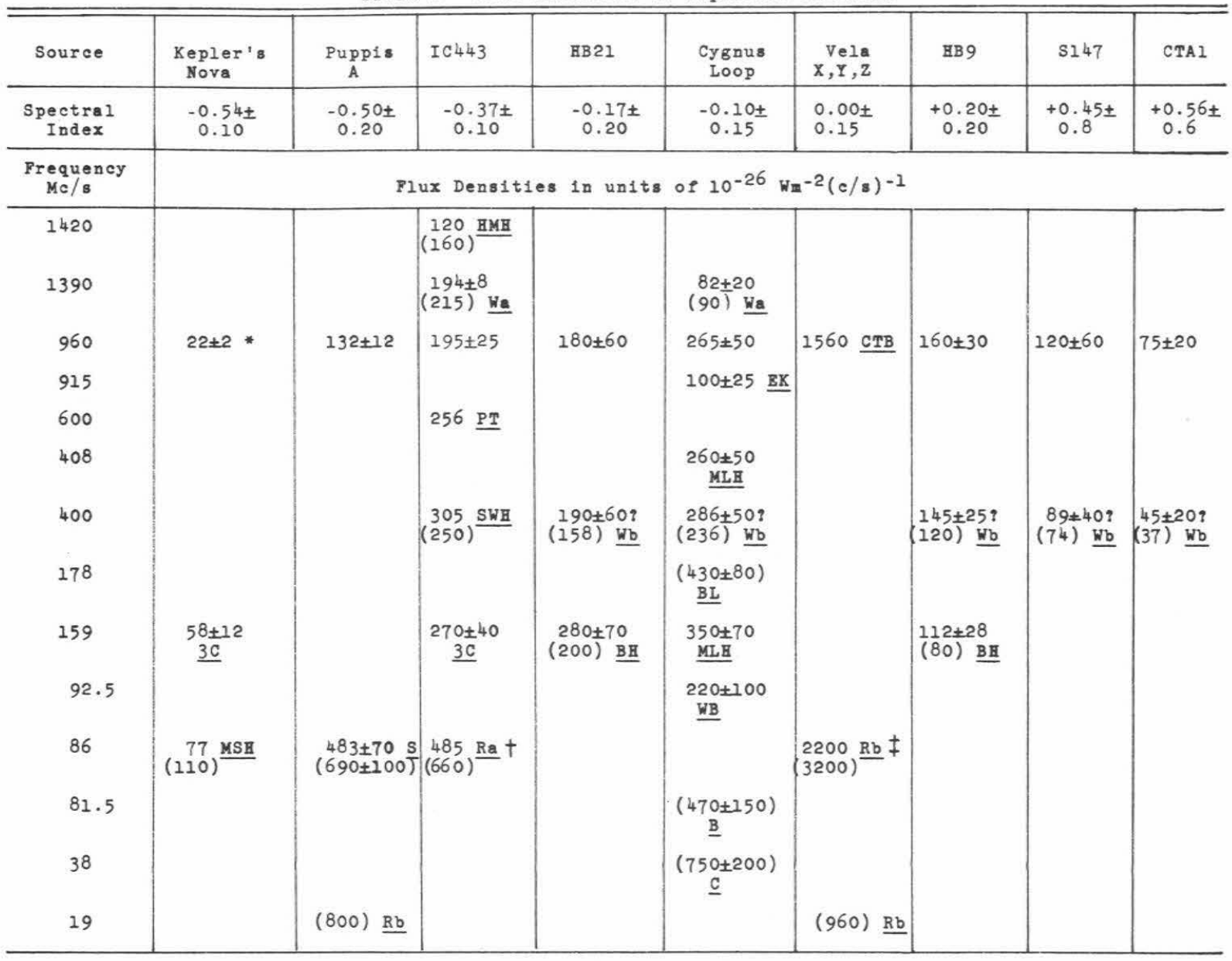

Parentheses indicate published flux densities which have not been normalized to the standard spectrum of M87.

? Indicates the error 18 the present author's estimate.

* CTA value corrected for pointing error.

+ Normalized to M87 by use of MS value of M87 and the Crab Nebula.

F Rishbeth gives an integrated flux density for Vela $x$, but only peak antenna temperature for Vela $Y$ and $Z$. The value of 3200 is the present author's estimate of the total flux density, which becomes 2200 after normalization (described in the text). The lover point shown on Figure 2 is the normalized intensity when only the peak values of Vela $Y$ and $Z$ are added to the 1ntegrated intensity of Vela $X$.

B - Baldwin (1955)

BH - Brown and Hazard (1953)

BL - Baldwin and Leslie (1960)

C - Costain (reported by BL)

CTB - Wilson and Bolton (1960)

EK - Eaton and Kraus (1959)

HMH - Hagen, McClain, and Hepburn (1954)

MLB - Mathevson, Large, and Haslam (1961)

MS - Mills and Slee (1957)

MSH - Mills, Slee, and Hill (1960)

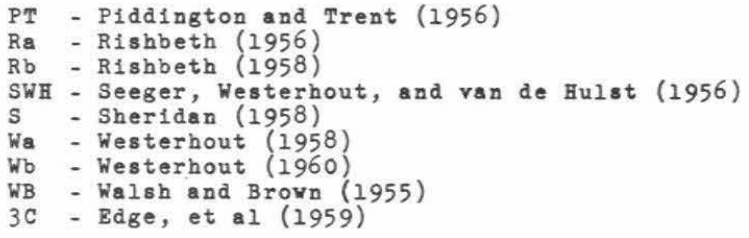




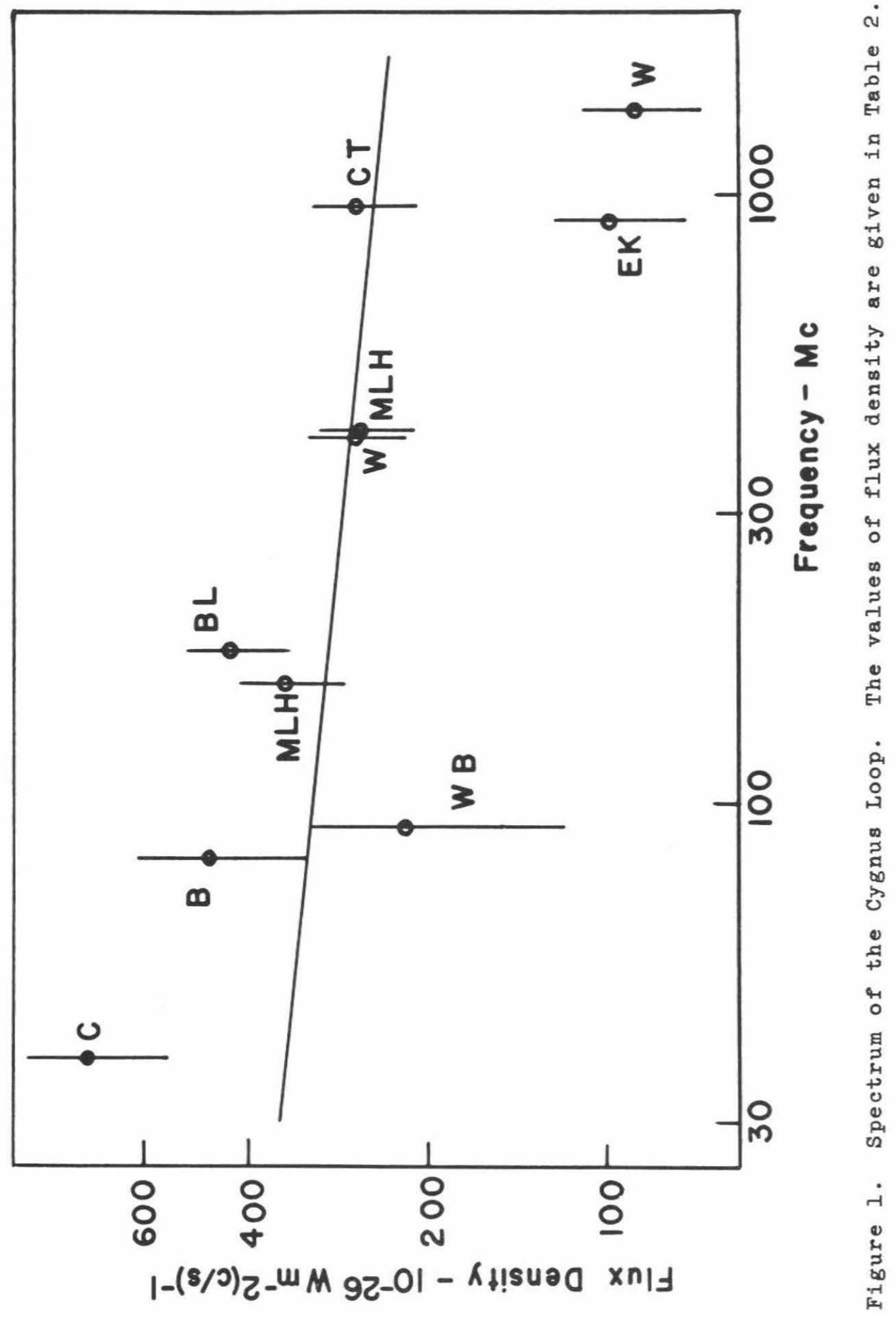


Table 3

Spectral Index of the 3 Parts of the Cygnus Loop

\begin{tabular}{|c|c|c|c|c|}
\hline Item & $\begin{array}{c}\text { Frequency } \\
(\mathrm{Mc} / \mathrm{s})\end{array}$ & $\underset{A}{\text { Source }}$ & $\underset{B}{\text { Source }}$ & $\underset{C}{\text { Source }}$ \\
\hline \multirow[t]{2}{*}{$\alpha(1950)$} & 960 & $20^{\mathrm{h}} 48^{\mathrm{m}} \cdot 4 \pm 0.3$ & $20^{h} 46^{m} \cdot 6$ & $20^{\mathrm{h}} 53^{\mathrm{m}} \cdot 3$ \\
\hline & 408 & $2048.3 \pm 0.3$ & 2046.7 & 2053.3 \\
\hline \multirow[t]{2}{*}{$\delta(1950)$} & 960 & $+29^{\circ} 52^{\prime} \pm 5^{\prime}$ & $+31^{\circ} 27^{\prime}$ & $+31^{\circ} 37^{\prime}$ \\
\hline & 408 & $+2940 \pm 5$ & +3128 & +3128 \\
\hline \multirow{3}{*}{$\begin{array}{l}\text { Integrated } \\
\text { Intensity } \\
\text { (percent of } \\
\text { total) }\end{array}$} & 960 & $64 \%$ & $25.8 \%$ & $10.2 \%$ \\
\hline & 408 & $62 \%$ & $28 \%$ & $9.7 \%$ \\
\hline & 178 & & & $9.3 \%$ \\
\hline \multirow{2}{*}{$\begin{array}{l}\text { Spectral } \\
\text { Index, rela- } \\
\text { tive to that } \\
\text { for entire } \\
\text { Loop }\end{array}$} & $960-408$ & +0.03 & -0.09 & +0.06 \\
\hline & $960-178$ & & & +0.05 \\
\hline
\end{tabular}

\section{c) Spectral Indices of Other Remnants}

The spectral indices listed in Table 5 (Appendix II) are based on the values of the CTA list unless supplementary information would change the spectral index significantly. For the Crab Nebula, a value of -0.25 is adopted. This is an average between the CTA value of -0.21 and the Dutch value (Woltjer 1958) of about -0.3. Flux densities for sources examined in more detail are fisted in Table 2 and plotted in Figure 2. The $960 \mathrm{Mc} / \mathrm{s}$ values listed for HB9, HB2l, and CTAl are derived from the contour diagrams given in Appendix III. (The quoted flux density for HB2l has had the galactic background subtracted.)

The spectra of $\mathrm{S} 147$ and CTAl are not well determined since they depend on only two points of low accuracy $(960$ and $400 \mathrm{Mc} / \mathrm{s})$. As in the case of the Cygnus Loop, the $400 \mathrm{Mc} / \mathrm{s}$ intensities of Westerhout were changed slightly in order to achieve a spectrum relative to that of M87. For HB9 and HB2l, flux densities are also available at $159 \mathrm{Mc} / \mathrm{s}$ (Hanbury Brown and Hazard 1953). An attempt to normalize the Brown and Hazard values to the $3 \mathrm{C}$ intensity scale was made by adopting a multiplying factor of 1.4 . This figure is the ratio $\mathrm{s}_{3 \mathrm{C}} / \mathrm{s}_{\mathrm{B} \& \mathrm{H}}$ for Cas $\mathrm{A}$. Finally, the $85 \mathrm{Mc} / \mathrm{s}$ flux densities taken with the Mills cross have been multiplied by 0.7 . This reduces the value of M87 published by Mills and Slee (1957) to that derived from our adopted spectrum of M87. 

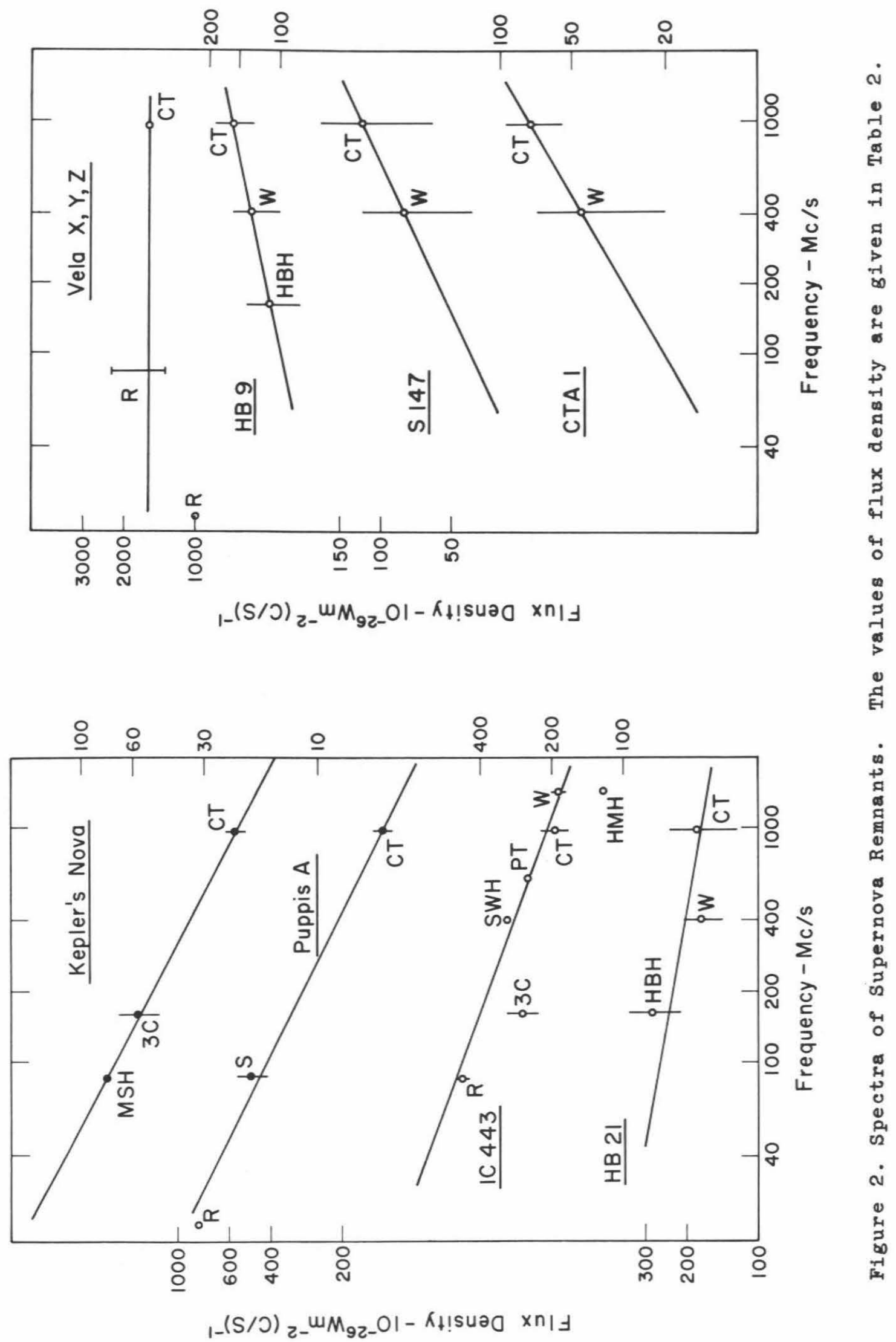
The slopes of the straight lines of Figure 2 are listed in Table 2 as well as in Table 5 (Appendix II). The quoted errors were estimated by fitting lines of different slopes to the plotted flux densities.

\section{Free-Free Emission}

In Harris and Roberts (1960), it was shown that most radio sources have spectral indices of $-0.62 \pm 0.25$. The notable exceptions to this rule are the galactic sources which may have flatter spectra with indices in the neighborhood of zero. Since this is the value of spectral index expected for optically thin HII regions, the occurrence of a nearly flat spectrum is often classified "thermal" with the implication that free-free transitions in ionized hydrogen are the source of the radiation (e.g. Wilson and Bolton 1960).

This line of reasoning has been extended to the Cygnus Loop by Mathewson, Large and Haslam (1961), who treat the spectrum of Source C as the combination of a "thermal" component with $x=0$ and a "nonthermal" component with $x=-0.5$. This interpretation was encouraged by the observation of MLH that the spectrum of the prominent optical region (Source C) was flatter than that of the prominent radio region (Source A). However, the comparison of the 960 and $408 \mathrm{Mc} / \mathrm{s}$ results (Figure 1 and Table 3) suggests that the spectrum of each part of the Loop is flatter than that found by MLH in the $159-408 \mathrm{Mc} / \mathrm{s} \mathrm{range}$. Therefore, it seems advisable to reconsider the importance of freefree emission in the various parts of the radio source.

From an order of magnitude count of filaments throughout the Cygnus Loop, it appears that Source A should have about one-half as much thermal emission as Source C. However, the integrations of Table 3 show that A contributes over six times more radio emission than does $\mathbf{C}$. Thus, even if Source C were entirely thermal, Source A should be $90 \%$ non-thermal. Furthermore, Figure 3 shows that the radio emission of Source A is centered well away from the main group of filaments. These considerations indicate that the nearly flat spectrum of the Cygnus Loop does not necessarily imply the existence of free-free emission.

The emission from Source $C$, however, is generally considered to be a combination of thermal and non-thermal radiation (Baldwin and Leslie 1960; MLH 1961). To derive an independent estimate of the importance of the free-free process in Source C, we have used optical measurements to calculate the expected radio emission (Appendix I). These calculations are based on Chamberlain's observations (1953b) of the surface brightness in $H \alpha$ of several filaments in the Cygnus Loop. It has been assumed that absorption of the H $\alpha$ emission by interstellar dust is negligible. Star counts made in selected regions extending from the Loop toward the galactic plane by Bok and Warwick (1957) seem to justify this assumption. Although their blue plates revealed 1 to 4 magnitudes of absorption at 500 or $1000 \mathrm{pc}$, the red ( $\lambda 6300)$ absorption was generally small, and in the immediate vicinity of the Loop, it was less than one-half magnitude.

The chief source of error in the calculation of expected radio emission is due to the uncertainty of the estimated area which is ap- 


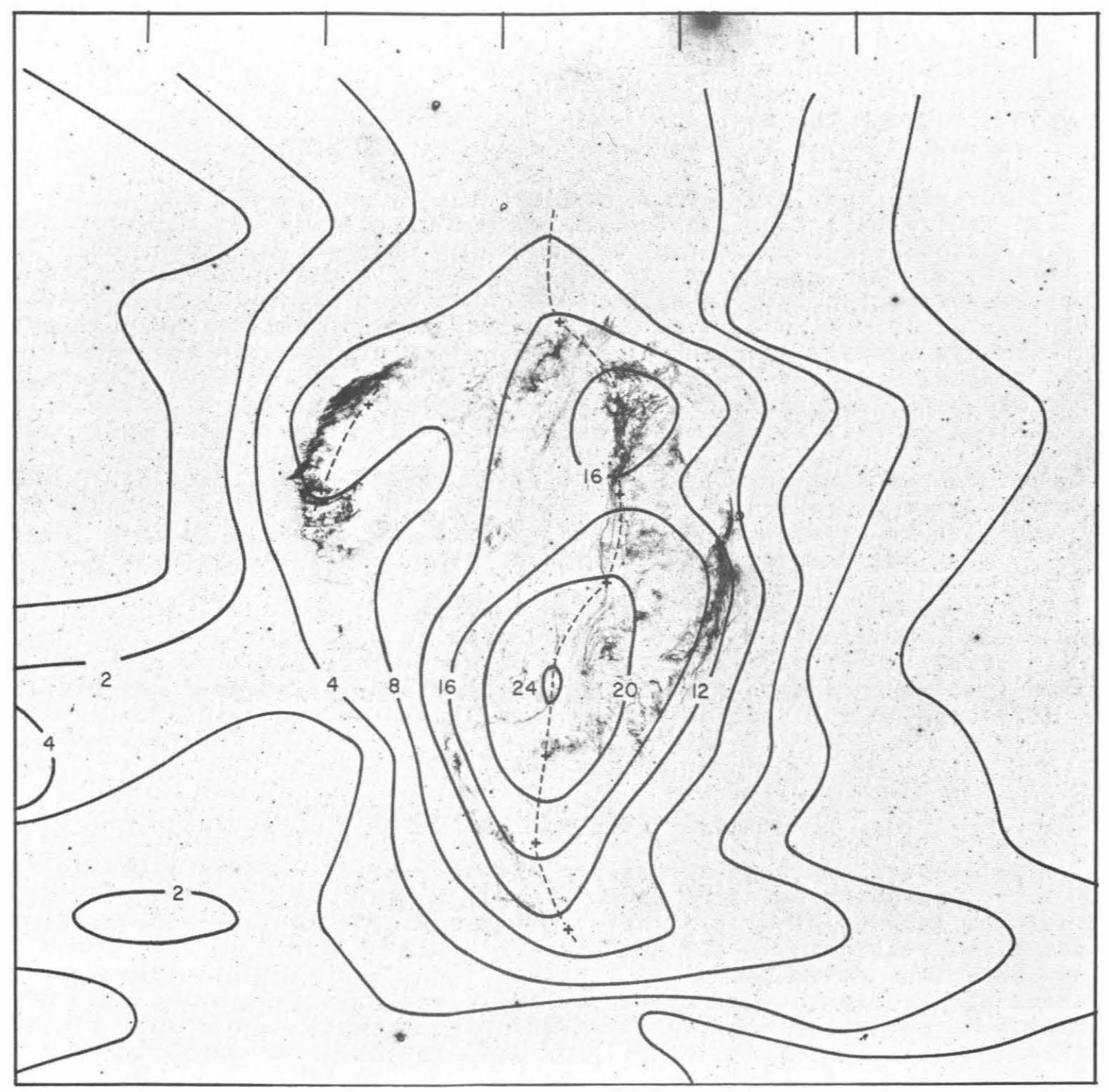

Figure 3. Comparison of the Radio and Optical Emission from the Cygnus Loop. Contours of antenna temperature (arbitrary scale) are the same as in Figure 6 . 
propriate for the surface intensity measurements of Chamberlain. We have taken the area as 250 filaments of size quoted by osterbrock (1958): $1.8 \times 4 ! 5$. This figure may contain an error of a factor of two. The calculations predict that $4 \%$ of the total radio emission from Source $C$ is contributed by free-free transitions in the filaments. An additional 2\% may come from a hypothetical sheet of lonized hydrogen which is undetected by even the deep red plates of the $48^{\prime \prime} \mathrm{Schmidt}$.

Appendix I also contains estimates of the free-free emission from HB9, where it has been assumed that the optical source is due to Ha emission. We have used the "plate limit" calculation of Appendix I, as HB9 is barely visible on the $E$ plate of the survey, but is quite evident on Minkowski's deep red plates (Figure 10). The calculations give an expected free-free emission equal to $2.5 \%$ of the total radio emission from HB9. Although there are two galaxies visible at the south west edge of the source, the most uncertain quantity in this calculation is the interstellar absorption. If two magnitudes of absorption were present, it would raise the computed $2.5 \%$ to $16 \%$.

A similar figure holds for $\mathrm{HB} 21$, which is even fainter optically and slightly brighter at $960 \mathrm{Mc} / \mathrm{s}$ than HB9. Furthermore, comparatively strong optical emission (presumably $H \alpha$ ) to the southwest of HB2l produces a barely detectable radio flux (compare Figures 8 and 11 ).

Assuming that the radiation from these objects is primarily nonthermal, a search for linear polarization would be pertinent. Such an attempt was made for the Cygnus Loop during November 1959. Unfortunately, the techniques used were not accurate enough to provide a positive detection of any polarized component less than $30 \%$ of the total radio emission.

\section{Empirical Spectral Index Variation}

In this section we propose the hypothesis that the spectra of supernova remnants flatten with age. This idea was suggested by the loose correlation of flatter-type spectra with increasing angular size which is apparent in Table 5 (Appendix II). Unfortunately, reliable age estimates are avallable for only a few objects. Therefore, we have chosen two physical parameters which assume the role of age indicators: the linear diameter and the radio surface brightness. Although neither of these variables can be expected to maintain a strict one-toone correspondence with age, the correlation should be satisfactory if each type of supernova forms a homogeneous group.

\section{a) The Linear Diameter as a Time Parameter}

The large uncertainties in our knowledge of diameters follows directly from inadequate values of the distance to the remants. Reliable distances are available for only Cas A, the Cygnus Loop, and the Crab Nebula. An order of magnitude estimate of other distances may be obtained from both optical and radio observations.

(i) Optical Methods of Distance Determination. We introduce the assumption that the physical diameter of structural details does not vary greatly from remnant to remnant. This assumption appears satisfactory for the three sources of known distance. We differentiate between the 
long thin filaments prominent in the Cygnus Loop and the thicker knots of Cas A. Knots also occur in HB2l, in Pup A, and in the south-west section (Source A) of the Cygnus Loop. The knots of Source A are about 3 times thicker than filaments, but are about the same size as the knots in Cas $A$.

The measurements which determine the filament sizes are given in Table 4. Unfortunately, high resolution plates were not available for all the sources and angular diameter measurements were often made from the 48-inch Schmidt plates. This method suffers from the lack of resolving power inherent in the small scale of the schmidt telescope and the use of Schmidt plates is therefore limited to objects closer than 2 kpc. Distances computed from filament sizes are listed in Table 5 (Appendix II).

Table 4

Filament Diameters

\begin{tabular}{|c|c|c|c|}
\hline Source & $\begin{array}{c}\text { Diameter } \\
\text { (sec.of arc) }\end{array}$ & $\begin{array}{l}\text { Distance } \\
(p c)\end{array}$ & $\begin{array}{l}\text { Diameter } \\
(p c)\end{array}$ \\
\hline $\begin{array}{l}\text { Cas A } \\
\text { Knots }\end{array}$ & 1 to 2 & 3400 & 0.016 to $0.033^{*}$ \\
\hline $\begin{array}{l}\text { Crab Nebula Filaments } \\
\text { Faint, thin } \\
\text { Bright, thick }\end{array}$ & $\begin{array}{l}1.6 \pm 0.6 \\
4.6 \pm 0.6\end{array}+$ & 1100 & $\begin{array}{l}0.0085 \pm 0.003 \\
0.024 \pm 0.003\end{array}$ \\
\hline $\begin{array}{l}\text { Cygnus Loop } \\
\text { Filaments } \\
\text { Knots }\end{array}$ & $\begin{array}{c}1.8 \neq \\
5.0^{1.8 \pm 1.8 \xi}\end{array}$ & 770 & $\begin{array}{l}0.0067 \\
0.018 \pm 0.006\end{array}$ \\
\hline $\begin{array}{l}\text { Adopted Size } \\
\text { Filaments } \\
\text { Knots }\end{array}$ & & & $\begin{array}{l}0.007 \\
0.02\end{array}$ \\
\hline
\end{tabular}

The $t$ values are an indication of the range of measurements for different filaments.

* Minkowski (1959).

tMeasured from reproduction of Baade's 200" plate (1956).

Fosterbrock's measurement of 100" plate (1958).

$\S 48^{\prime \prime}$ Schmidt print. 
On the basis of this estimate of distance, the diameter of each remnant has been computed and plotted against the spectral index (Figure 4). The open circles represent objects for which distances were derived from optical measurements. The constant filament size hypothesis was used for all objects except Cas A, the Crab Nebula, Kepler's and Tycho's supernovae, and the Cygnus Loop. Distances of these sources were inferred from radial velocity and proper motion observations (Minkowski 1958, 1959).

Most of the plotted points lie in the neighborhood of a line constructed through Cas A and the Cygnus Loop. This line is tentatively identified as the path taken by evolving type II remnants. Type I remnants such as the Crab Nebula, and possibly Tycho's Nova, Kepler's Nova, and CTAl, might follow a similar path characterized by much smaller diameters. This interpretation of Figure 4 would suggest that a supernova remnant forms with a steep spectrum and small diameter. As it evolves, its path would stay close to the line drawn: the spectral index becoming less negative as the remnant expands.

Since the spectral index would not be expected to change without limit, an exponential curve has been fitted to Cas A and the Cygnus Loop. As $r$ approaches infinity, we have let the spectral index approach +0.5. The asymptotic behavior for $r$ close to zero has not been fixed, as we have no knowledge of the spectral index for very young remnants. The equations associated with type II supernova are then found to be:

$$
\begin{array}{ll}
x=0.765(\log d)-1.26 & \text { straight line fit } \\
x=-2.11 d^{-0.36}+0.5 & \begin{array}{l}
\text { exponential fitted to the form: } \\
x=a \cdot \exp (-b \cdot \log d)+0.5 .
\end{array}
\end{array}
$$

For future reference, equation (2) may be expressed as:

$$
r(\text { parsecs })=172 \beta^{-2.74}
$$

where

$$
\begin{aligned}
& \mathrm{d}=\text { diameter (parsecs) } \\
& r=\text { radius (parsecs) } \\
& \mathrm{x}=\text { spectral index } \\
& \beta=2 \gamma=2(1-2 \mathrm{x})
\end{aligned}
$$

(ii) Radio Method of Distance Determination. Shklovsky has recently published two papers on supernova remnants (1960a, 1960b). In the first paper, he has formulated an expression for the radio emission of a remnant as a function of size, and in the second he applies this principle to obtain the distance of particular remnants. The basic assumptions of Shklovsky's approach are:

(a) Synchrotron radiation is responsible for the observed radio emission.

(b) Initial conditions do not vary significantly from remnant to remnant. For this reason, the discussion is restricted to type II remnants. 


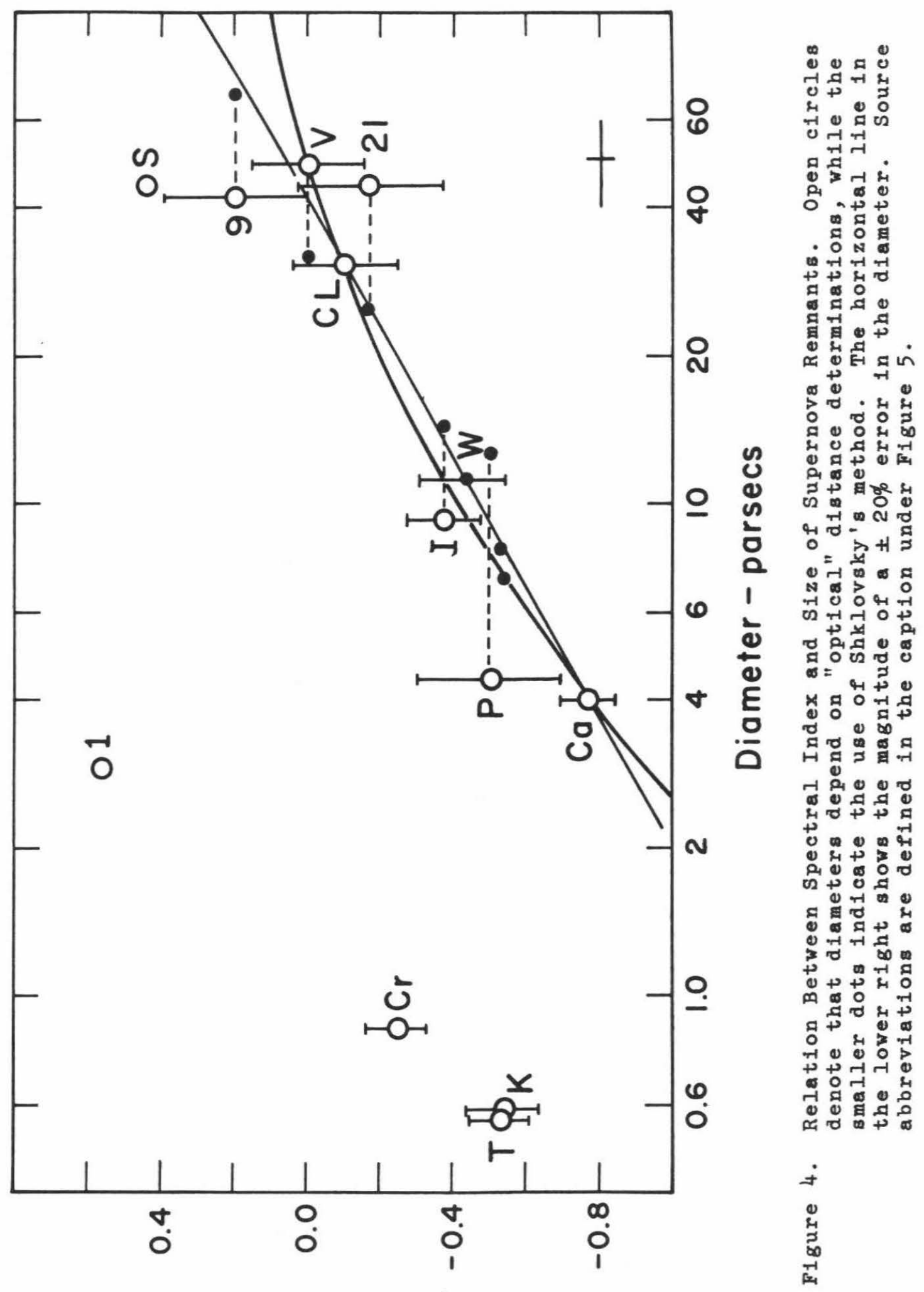

xәрu| |Dגtords 
(c) The magnetic field strength is given by $H=H_{0}\left(r_{0} / r\right)^{2}$.

(d) The energy of the relativistic electrons is degraded chiefly by the expansion of the remnant: dE/dt $=-(V / r) E$. From this, Shklovsky derives $K=K_{0}\left(r_{0} / r\right)^{\gamma}-1\left(r_{0} / r\right)^{3}$.

$\mathrm{K}$ is the amplitude of the electron energy distribution: $\mathrm{dN}(\mathrm{E})=\mathrm{KE} \mathrm{E}^{-\gamma} \mathrm{dE}$.

$r_{0}$ is the radius at some initial time.

$K_{O}$ and $H_{O}$ are $K$ and $H$ evaluated at that time.

$\nabla$ is the velocity of expansion of the remnant.

$E$ is the energy of an electron.

The power emitted by a remnant due to the synchrotron mechanism is given to sufficient accuracy (Shklovsky 1960a) by:

$$
F_{v} \propto\left(2.8 \cdot 10^{8}\right)^{\frac{\gamma-1}{2}} \mathrm{r}^{3} \mathrm{~K} \mathrm{H}_{\perp}^{\frac{\gamma+1}{2}} v \frac{1-\gamma}{2}
$$

When $\mathrm{K}$ and $\mathrm{H}$ are replaced by the expressions given above, the surface intensity (rather than the emission of the whole nebula) becomes:

or

$$
\begin{aligned}
& {\left[\frac{\mathrm{S}_{\nu}}{\phi^{2}}\right] \alpha\left[\frac{\mathrm{F}_{\nu}}{4 \pi \mathrm{r}^{2}}\right] \propto\left\{\mathrm{H}_{0} \mathrm{~K}_{0} \mathrm{r}_{\mathrm{o}}^{3}\left[\frac{2.8 \cdot 10^{8} \mathrm{H}_{\mathrm{o}}}{\nu}\right]_{\text {where }}^{-1 / 2}\right\}\left\{\left[\frac{2.8 \cdot 10^{8} \mathrm{H}_{\mathrm{o}}}{\nu}\right]^{1 / 4} \mathrm{r}_{0}\right\}^{\beta} \mathrm{r}^{-(\beta+2)}} \\
& \mathrm{S}_{\nu} / \phi^{2}=\mathrm{A}_{\nu} \mathrm{B}_{\nu}^{\beta / \mathrm{r}^{\beta+2}} \quad \mathrm{~B}_{\nu}=\left(2.8 \cdot 10^{8} \mathrm{H}_{\mathrm{o}} / \nu\right)^{1 / 4} \mathrm{r}_{\mathrm{o}}
\end{aligned}
$$

$S_{v}=$ flux density arriving at the earth.

$\varnothing=$ angular radius of the remnant.

$R$ = distance to the remnant.

Shklovsky then substitutes $r=R \varnothing$ to calculate the distance from the observed surface intensity and angular size. Unfortunately, individual values of spectral index were not avalable to Shklovsky and he assumed that all the older remnants had a spectral index of -0.5 . Thus he was able to lump the constants $A_{v}$ and $B_{v} \beta$ together and use only the Cygnus Loop as the normalizing condition. With the present values of spectral index, equation (4) has been used to recalculate distances where the Cygnus Loop and Cas A serve as the conditions necessary to determine $A_{y}$ and $B_{y}$. On the basis of these revised distances, diameters have been computed and these values are shown as filled circles in Figure 4.

The question arises as to how sensitive the calculated distances are to assumptions (c) and (d). To answer this question in part, dis tances were computed when either (c) was replaced by $H=H_{0}\left(r_{0} / r\right)$ or (d) was replaced by $d E / d t=-$ const (the approximate case for ionization losses). These changes varied the calculated distances for the type II remnants by $40 \%$ or less, and therefore the only values of diameter given are those based on the original assumptions. 


\section{b) The Radio Surface Brightness as a Time Parameter}

Another approach to the proposed time variation of spectral index is possible if the surface brightness is assumed to decrease with age. Figure 5 is a plot of a source's surface intensity at $960 \mathrm{Mc} / \mathrm{s}$ against 1ts spectral index. The surface intensity errors shown were derived from flux density errors and estimated errors of the radio diameter. The line drawn in Figure 5 demonstrates the behavior which might be expected of a remnant from considerations of the previous section. This line is a plot of equation (4) where $r$ has been replaced by the use of equation ( $2 a)$ :

$$
\log \frac{S_{\nu}}{\phi^{2}}=\log A_{\nu}+\beta \log B_{\nu}-(\beta+2) \log \left[\frac{172}{\beta^{2.74}}\right]
$$

As noted previously, even though the exact form of equation (4) depends on the assumptions (c) and (d), the general behavior of the surface intensity will be approximated by equation (5).

Although the plotted points of Figure 5 do not lie exactly along the line, they are consistent with the expectation of low surface intensity occurring with flat spectra. This correlation, which does not rely on a knowledge of the remnant's distance, tends to support the inference of Figure 4.

An interesting feature of Figure 5 is the apparent similarity of the surface intensity of Kepler's and Tycho's supernova remnants to that of the type II remnants rather than to that of the Crab Nebula (see also Biraud, Lequeux and LeRoux 1960). Moreover, if the distances to these remnants are computed by the "revised Shklovsky method", which is calibrated by Cas $A$ and the Cygnus Loop, the resulting diameters satisfy equation (2) for type II remnants (the two unlabelled points near Pup A on Figure 4). The distances required for this interpretation are listed in parentheses in Table 5 but seem unreasonably large when a moderate amount of optical absorption is assumed. In any event, the seeming discrepancy between the radio and optical observations may be resolved when the differences, if any, between the radio surface brightnesses of type I and type II remnants is more fully understood.

\section{c) Remarks}

The physical explanation of the proposed spectral index variation poses a difficult problem. The well-known relation between the spectral index of the radiation, $x$, and the exponent of the electron energy dis tribution, $Y$, is given by $Y=(1-2 x)$. Thus, when a power law of the form $N=$ const $E-\gamma$ is assumed for the number of electrons with energy, $E$, the spectral index of the radio emission uniquely determines $r$. If the radio spectrum does flatten with age, the energy distribution of the electrons responsible for this radiation must alter in the sense of an ever decreasing relative number of low energy electrons. Although we have no completely satisfactory explanation for this behavior, two alternatives have been considered: a selection effect which would separate particles of different energies; and a temporal effect which would modify the energy spectrum by a change of the energy loss mechanism for the relativistic electrons. 


\section{Spectral Index}

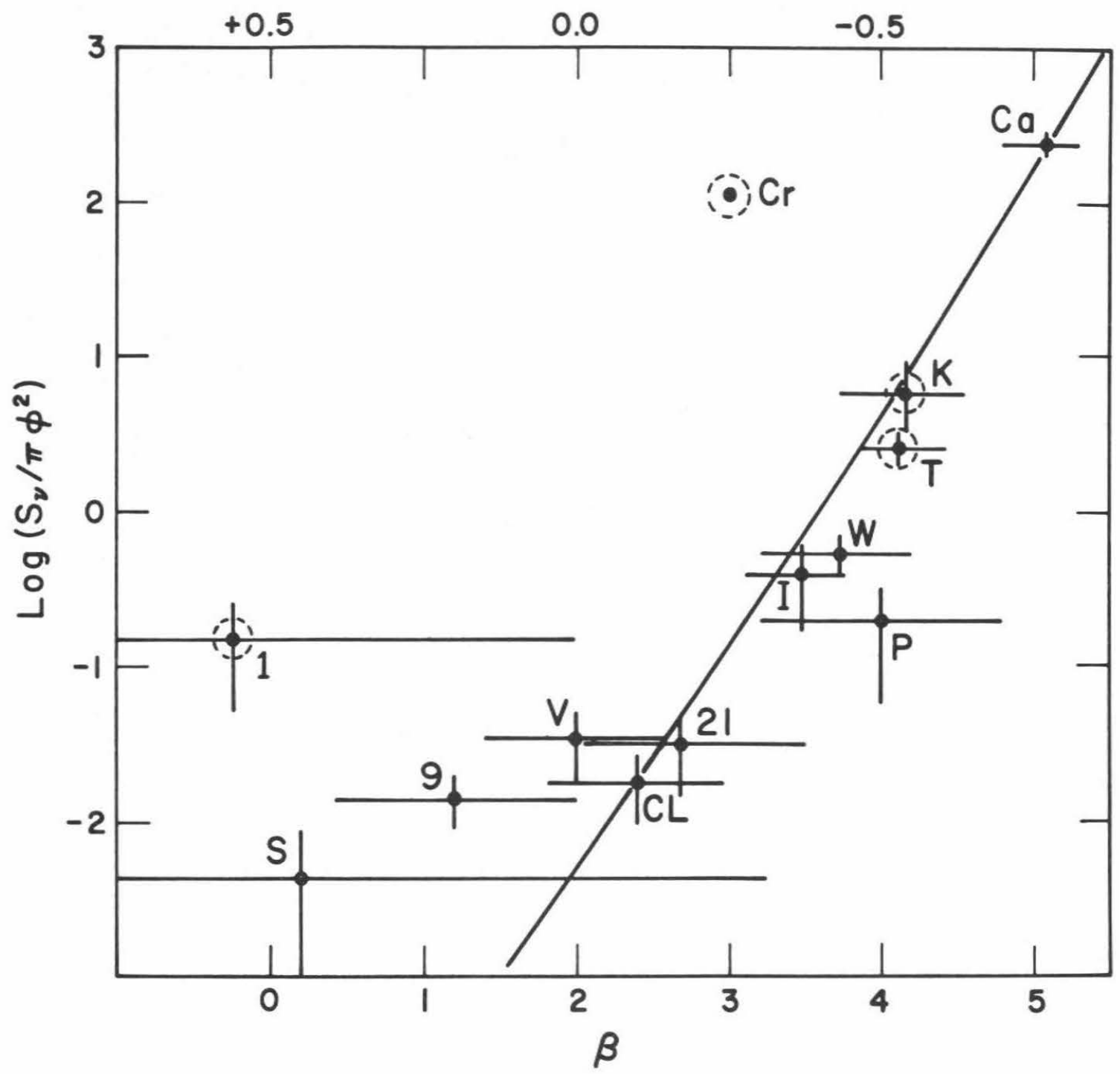

Figure 5. Relation Between Spectral Index and Surface Brightness of Supernova Remnants. The unit of surface brightness is $10^{-26} \mathrm{Wm}^{-2}(\mathrm{c} / \mathrm{s})-1 / \square^{\prime}$. Type I remnants are encircled by dotted lines.

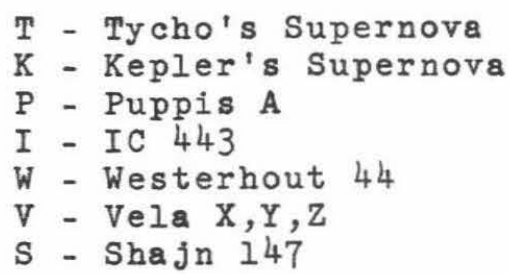

T - Tycho's Supernova

K - Kepler's Supernova

P - Puppis A

I - IC 443

$\mathrm{V}$ - Vela X,Y,Z

S - Shajn 147

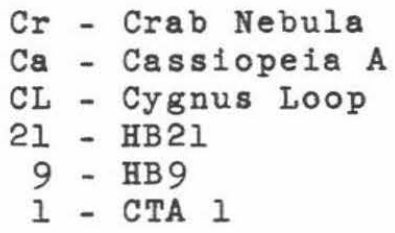


The latter of these two possibilities seems to be the most feasible. Although a quantitative treatment has not been succesful, a qualitative description of how the physical parameters might allow a suitable change in energy loss mechanism is outlined in the author's thesis (Harris 1961 ).

\section{Discussion}

\section{a) Association of Supernova with Early-Type Stars}

The possible confusion between HII regions and supernova remnants will become especially interesting if hybrid objects exist. Öpik (1953) and, more recently, Shklovsky (1960b) have discussed the probability that some supernova remnants should occur in $O B$ associations since the most massive (and hence most rapidly evolving) stars are often found there. Shklovsky has suggested that the Vela X complex may be an example of this, and also that the non-thermal emission from the Orion Nebula region could be due to an old supernova remnant. One is tempted to add the Rosette Nebula to this list. This nebula has a well defined shell structure and could prove to be a hybrid object which exhibits nonthermal radiation together with free-free emission from hydrogen ionized by nearby early-type stars.

\section{b) Spectral Index Variation}

If the remnants do follow the evolutionary line of Figure 4 rather than expanding with constant spectral index, there should be a secular decrease of spectral index which would be most noticable for young, rapidly expanding remnants. From equation (2) the annual change of spectral index for Cas A is calculated to be 0.0015 . For the Cygnus Loop the expected change is $10^{-6}$ per year. Although these changes are very small, in twenty years the spectral index of Cas A should change by 0.03. Extrapolating back in time for Cas A yields a lower limit of $\Delta x=+0.3$ for 200 years. At that time, Cas $A$ would have been at the steep end of the spectral index distribution of all radio sources.

The changing spectral index will also make a small correction to Shklovsky's calculation of the rate of emission decay. He predicts a secular decrease of the flux density of $2 \%$ a year for Cas A. A recalculation of this quantity, which includes a changing spectral index, gives about $1.3 \%$ a year: a value not significantly different from the original prediction.

Shklovsky has argued that the surface brightness of remnants with steep spectra will decrease more rapidly than that of remnants with flatter type spectra. This follows from the dependence of the emission on $H^{(\gamma+1) / 2}$ and the assumption that $\mathrm{H}=\mathrm{H}_{\mathrm{O}}\left(\mathrm{r}_{\mathrm{O}} / \mathrm{r}\right)^{2}$. Since observational selection will discriminate against sources of low surface brightness, one should find a correlation between flat spectra and old remnants even if the spectral index of each remnant does not change with time. This process would be most effective in reducing the population of sources lying to the right of the line in both Figure 4 and Figure 5 (steep spectra together with large diameter or low surface intensity). However, this selection should not discriminate against plat spectra 
concomitant with small diameter or high surface intensity (significantly to the left of either line). The observations to date do not place any type II remnants in these regions. Therefore, the absence of small, bright objects with flat spectra is the only empirical evidence which supports the time variation of spectral index, rather than Shklovsky's interpretation of a fixed spectral index.

The distinction between these alternative suggestions is a particular example of the rather general concept that any natural spread in the value of a physical parameter (of a group of objects) is usually caused either by a variation of initial conditions or by age differences in an evolutionary sequence. Both mechanisms are likely to be operative in any given situation, but often only one will dominate. Thus, Shklovsky's description of supernova remnants would ascribe the spread in spectral indices to a variation of initial conditions, while the interpretation proposed here is that each remnant forms with nearly the same value of spectral index, but this value changes with time.

\section{c) Conclusion}

If the synchrotron process is responsible for the radio emission of most sources, the variation of spectral index implies a variation in the energy distribution of the relativistic particles in the sources. We may expect that this distribution will depend on some other physical parameter, which in turn may be thought of as reflecting either an initial variation or an age variation. We suggest that the spectral index distribution of supernova remnants illustrates the domination of age dependent parameters and that the situation for the extragalactic sources may be similar.

\section{Acknowledgments}

It is a pleasure to thank Gordon Stanley for his critical reading of this manuscript and his many helpful suggestions. I would also like to express my gratitude to the staff and graduate students for their help in obtaining the observations and to R. Minkowski for his comments on the optical features of the sources and for the deep red plates reproduced in Appendix III. Much of this paper would have been impossible without access to the Manchester work on the Cygnus Loop and I am indebted to D. Mathewson for sending that in advance of publication. Special thanks are also due $G$. Westerhout for the $400 \mathrm{Mc} / \mathrm{s}$ flux densities of several sources. Finally, I would like to acknowledge the Consultants Bureau Inc., who kindly provided pre-publication translations of Shklovsky's papers. The observations reported here were carried out under Office of Naval Research Contract Nonr 220(19). 


\section{APPENDIX I}

\section{Calculation of Expected Free-Free Radio Emission}

a) Source $C$ of the Cygnus Loop

This calculation of free-free emission is based on the observed H $\alpha$ surface brightness measured by Chamberlain (1953b). In order to find the ratio of free-free emission in the radio continuum to the Ha line emission, we make the usual assumptions about conditions in the filaments :

1) the optical depth is small at $1000 \mathrm{Mc} / \mathrm{s}$ and also in the Ha line, but large for Lyman line radiation;

2) the Rayleigh-Jeans approximation of the Planck function is valid for computing the free-free emission at $1000 \mathrm{Mc} / \mathrm{s}$;

3) the electron temperature is $40,000^{\circ} \mathrm{K}$ and the electron density is constant throughout the emitting volume;

4) collisional excitation is responsible for the ionization (if radiative excitation predominated, $b_{n}$ would be lowered and the radio emission would be larger by a factor of about 3). Chamberlain (1953a)

Under these assumptions, the volume emissivity for free-free emission is (Osterbrock and Stockhausen 1960):

$$
\begin{aligned}
j_{1000}=4.6 \cdot 10^{-37} & \mathrm{~N}_{e^{N}} \mathrm{~N}_{1} \mathrm{~T}^{-1 / 2} \text { ergs } \mathrm{cm}^{-3} \mathrm{~s}^{-1}(\mathrm{c} / \mathrm{s})^{-1} \\
\text { where } \mathrm{N}_{e} & =\text { electron density } \\
\mathrm{N}_{1} & =\text { ion density } \\
\mathrm{N}_{\mathrm{p}} & =\text { proton density } \\
\mathrm{T}_{\mathrm{e}} & =\text { electron temperature }
\end{aligned}
$$

The volume emissivity of $H \alpha$ is taken as

$$
j_{H \alpha}=2.8 \cdot 10^{-18} \mathrm{~N}_{\mathrm{p}} \mathrm{N}_{\mathrm{e}} \mathrm{T}_{\mathrm{e}}^{-3 / 2} \text { ergs } \mathrm{cm}^{-3} \mathrm{~s}^{-1}
$$

For the surface intensity we have averaged the 3 values given by Chamberlain (1953b) for Source C filaments. This figure was then reduced by $56 \%$ to correct for the contribution of [NII] (Minkowski 1958). The resulting surface intensity is:

$$
\sigma_{\mathrm{H} \alpha}=14 \cdot 10^{-4} \text { ergs } \mathrm{cm}^{-2} \mathrm{~s}^{-1}
$$

Chamberlain has corrected for resolution, but no estimate of his error is given.

If there is no self absorption for the radio or Ha emission, then

$$
j_{1000} / j_{\text {H } \alpha}=\sigma_{1000} / \sigma_{\mathrm{H} \alpha}
$$


and from equations (6) and (7),

$$
\sigma_{1000}=10^{-20} \mathrm{Wm}^{-2}(\mathrm{c} / \mathrm{s})^{-1}\left(\text { for } \mathrm{N}_{i} / \mathrm{N}_{\mathrm{p}}=1.1\right. \text { ) }
$$

$\sigma 1000$ is the rate of energy flow per $\mathrm{m}^{2}$ in unit frequency interval at the surface of a filament. To obtain the brightness, or specific intensity:

$$
b_{1000}=\frac{\sigma_{1000}}{\pi}=3.2 \cdot 10^{-21} \mathrm{Wm}^{-2}(\mathrm{c} / \mathrm{s})^{-1} \mathrm{str} \cdot-1
$$

The surface of the filaments subtends a solid angle, $\wedge_{f}$ which is estimated by 250 filaments of size $1.8 \times 4: 5\left(n_{f}=2.8 \cdot 10^{-6}\right.$ str.).

The integrated flux density at the earth will then be:

$$
s_{1000}=n_{f} b_{1000}=9 \cdot 10^{-27} \mathrm{wm}^{-2}(\mathrm{c} / \mathrm{s})^{-1}
$$

or $4 \%$ of the observed $960 \mathrm{Mc} / \mathrm{s}$ flux density of Source C. It is felt that the chief source of error in this calculation enters from the uncertainty in correcting for the resolution of the telescope (24" Schmidt).

b) Free-Free Emission from Regions Optically Invisible

If the ionized hydrogen of the Cgynus Loop is in the form of sheets rather than filaments, one would expect that the apparently vacant areas could be sheets of ionized hydrogen seen face on. The emission measure would be too small to allow detection of the Ha radiation on the 48" plates as the path length would be very small. The following calculation gives an estimate of the flux density at the earth from free-free emission in ionized hydrogen which would be at the limit of detection on the $\mathrm{E}$ plates of the Sky Survey.

Abell (1960) quotes the following figures:

25.5 to 26 mag per square second of arc for the limit of visibility of the $E$ survey plates;

$2.004 \cdot 10^{32}$ ergs $s^{-1}$ for the total energy output of the sun contained in the spectral bandpass used;

-27.I as the apparent photo-red magnitude of the sun.

These figures reduced to surface brightness are:

and

$$
-27.1+2.510 g(\text { area of sun })=-11.0 \mathrm{mag} / \square^{\prime \prime}
$$

$\sigma_{\text {photo-red }}=\frac{\text { Energy of sun }}{\text { Area of sun }}=3.3 \cdot 109$ ergs $\mathrm{cm}^{-2} \mathrm{~s}^{-1}$

Therefore, the plate limit is $36.5 \mathrm{mag}$ fainter than the sun and, $\sigma$ photo-red (plate limit) $=8.3 \cdot 10^{-6}$ ergs $\mathrm{cm}^{-2} \mathrm{~s}^{-1}$

However, this figure needs a correction factor of 1.3 since the 
sensitivity curve of the E plates lowers $\lambda 6563$ (H $\alpha$ ) by $22 \%$ from the maximum of the band pass which was normalized on the sun. Therefore,

$$
\sigma_{H \alpha}(\text { plate limit })=10^{-8} \mathrm{~W} \mathrm{~m}^{-2}
$$

Using the ratio of surface brightnesses $\left(\sigma_{1000} / \sigma_{H \alpha}\right)$ computed earlier,

$$
\begin{aligned}
& \sigma_{1000}\left(\text { plate limit) }=7 \cdot 3 \cdot 10^{-23} \mathrm{wm}^{-2}(\mathrm{c} / \mathrm{s})^{-1}\right. \text { and } \\
& \mathrm{b}=\sigma / \pi=2 \cdot 3 \cdot 10^{-23} \mathrm{Wm}^{-2}(\mathrm{c} / \mathrm{s})^{-1} \mathrm{str} .^{-1} .
\end{aligned}
$$

For an area of two square degrees (roughly the area of Source C), the flux density at the earth will be:

$$
\mathrm{S}_{1000}=\mathrm{b} \Omega=1.4 \cdot 10^{-26} \mathrm{~W} \mathrm{~m}^{-2}(\mathrm{c} / \mathrm{s})^{-1}
$$

or $5 \%$ of the observed integrated flux density of Source $C$. On the deep red plates taken by Minkowski, this value may be lowered by a factor of 3 or 4 .

$$
\text { c) Free-Free Emission from HB9 }
$$

HB9 is barely visible on the E plates while it shows quite well on the deep red plates taken by Minkowski (Figure 10). The total area of HB 9 is about 6 square degrees or 3 times larger than the area of Source C, and therefore the flux density arriving at the earth would be

$$
\mathrm{S}_{\mathrm{HB} 9}=4.2 \cdot 10^{-26} \mathrm{~W} \mathrm{~m}^{-2}(\mathrm{c} / \mathrm{s})^{-1}
$$

or about $2.5 \%$ of the observed integrated flux density from HB9. This figure ignores the effect of interstellar absorption of the Ha line intensity. 


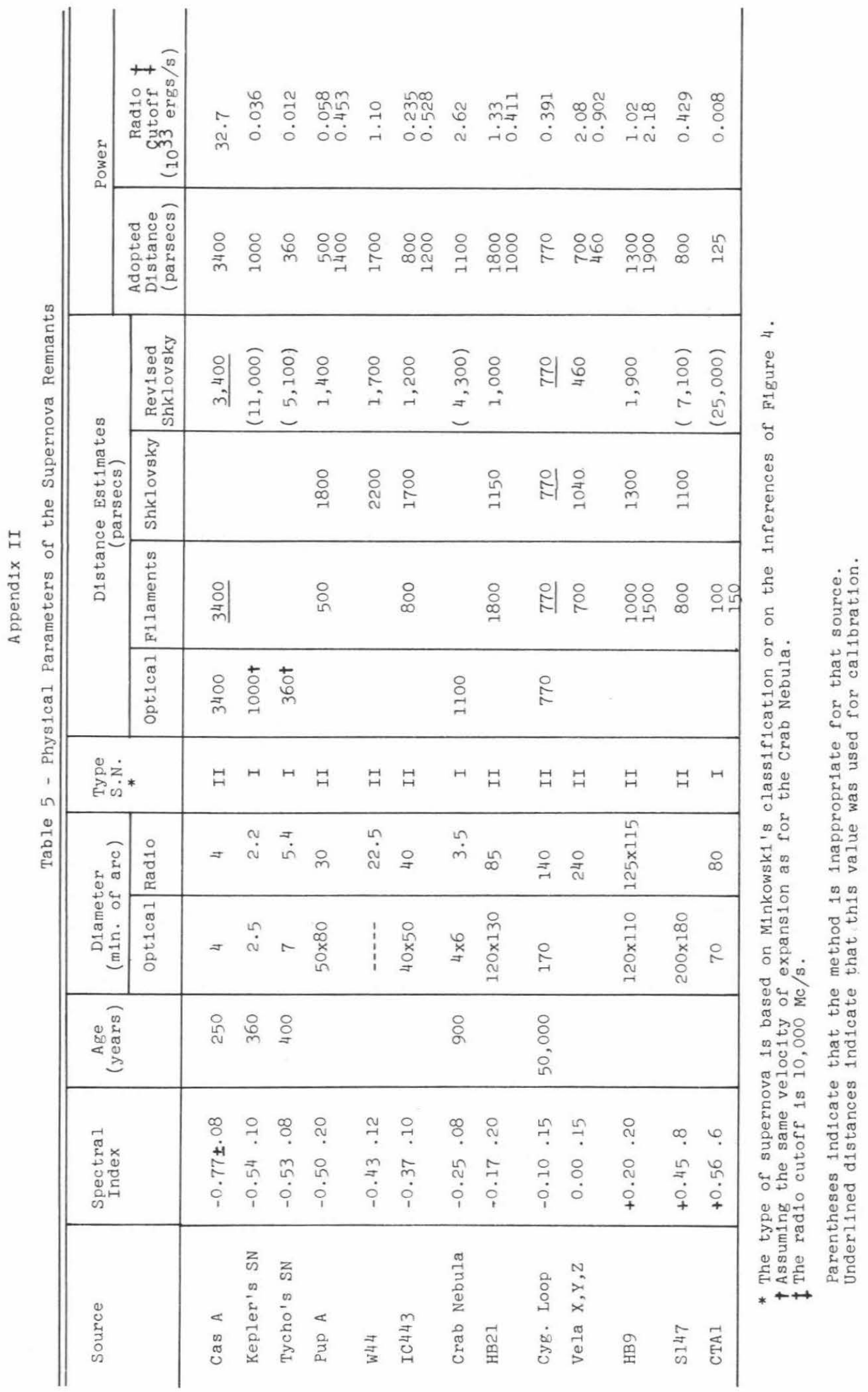




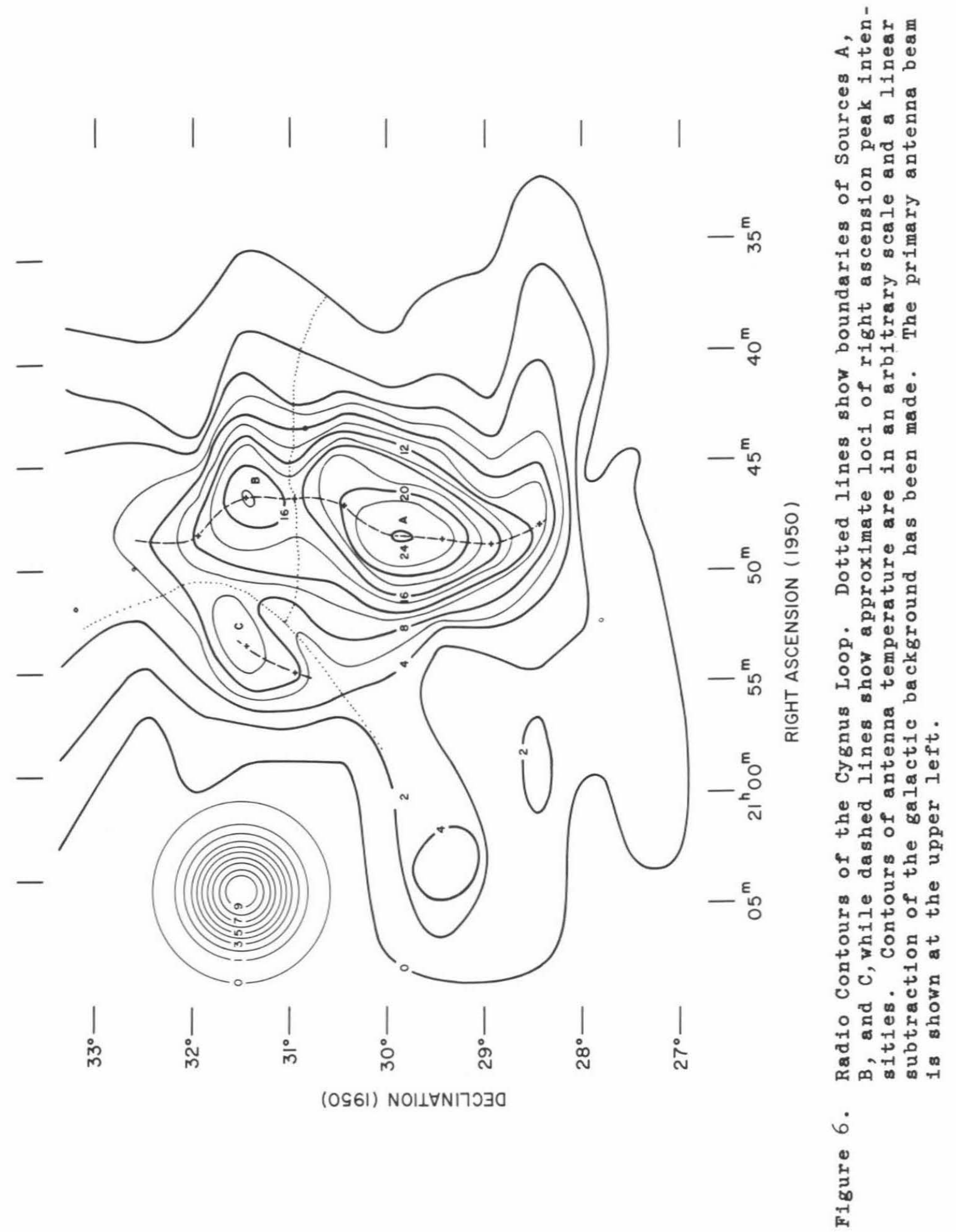




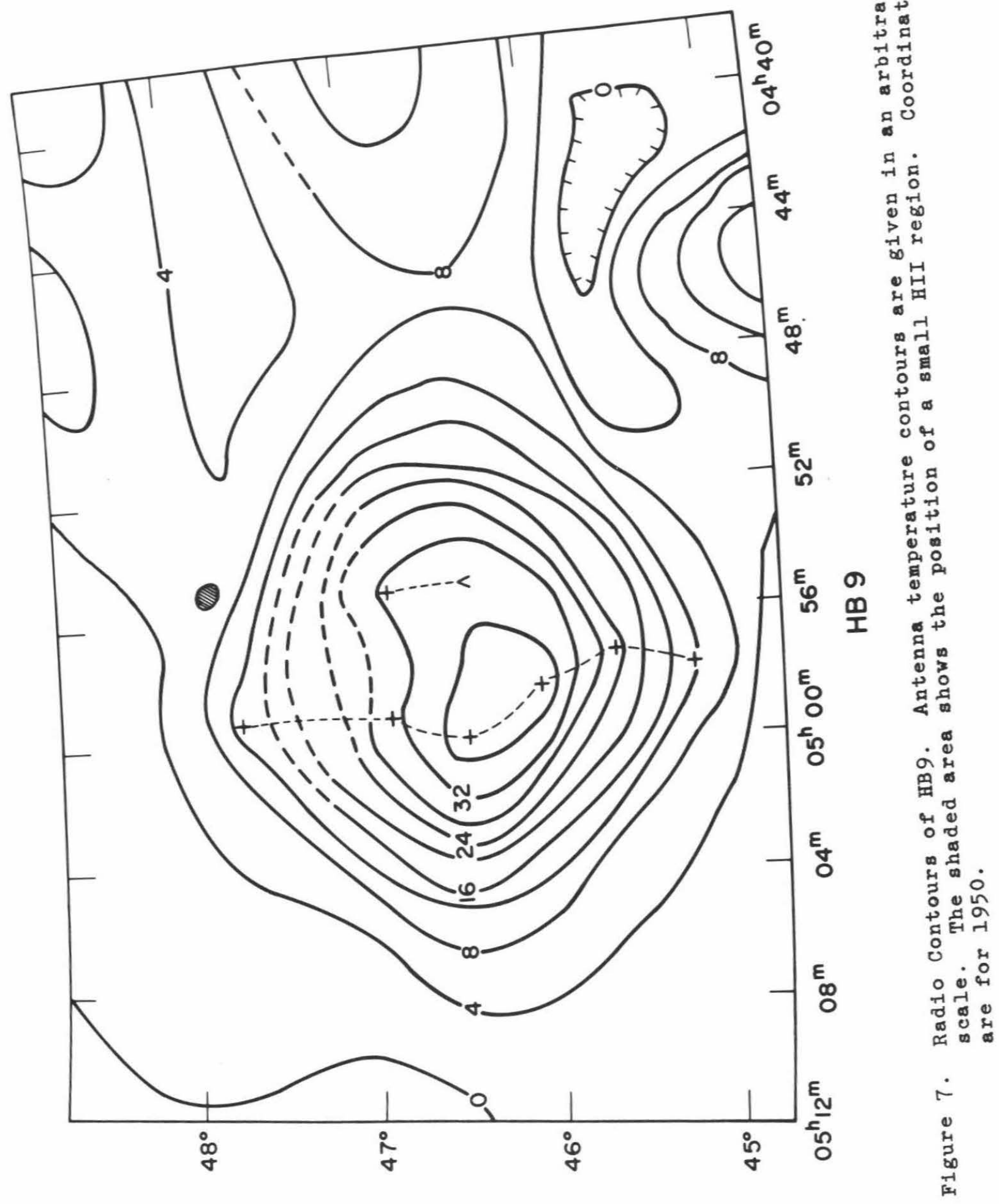




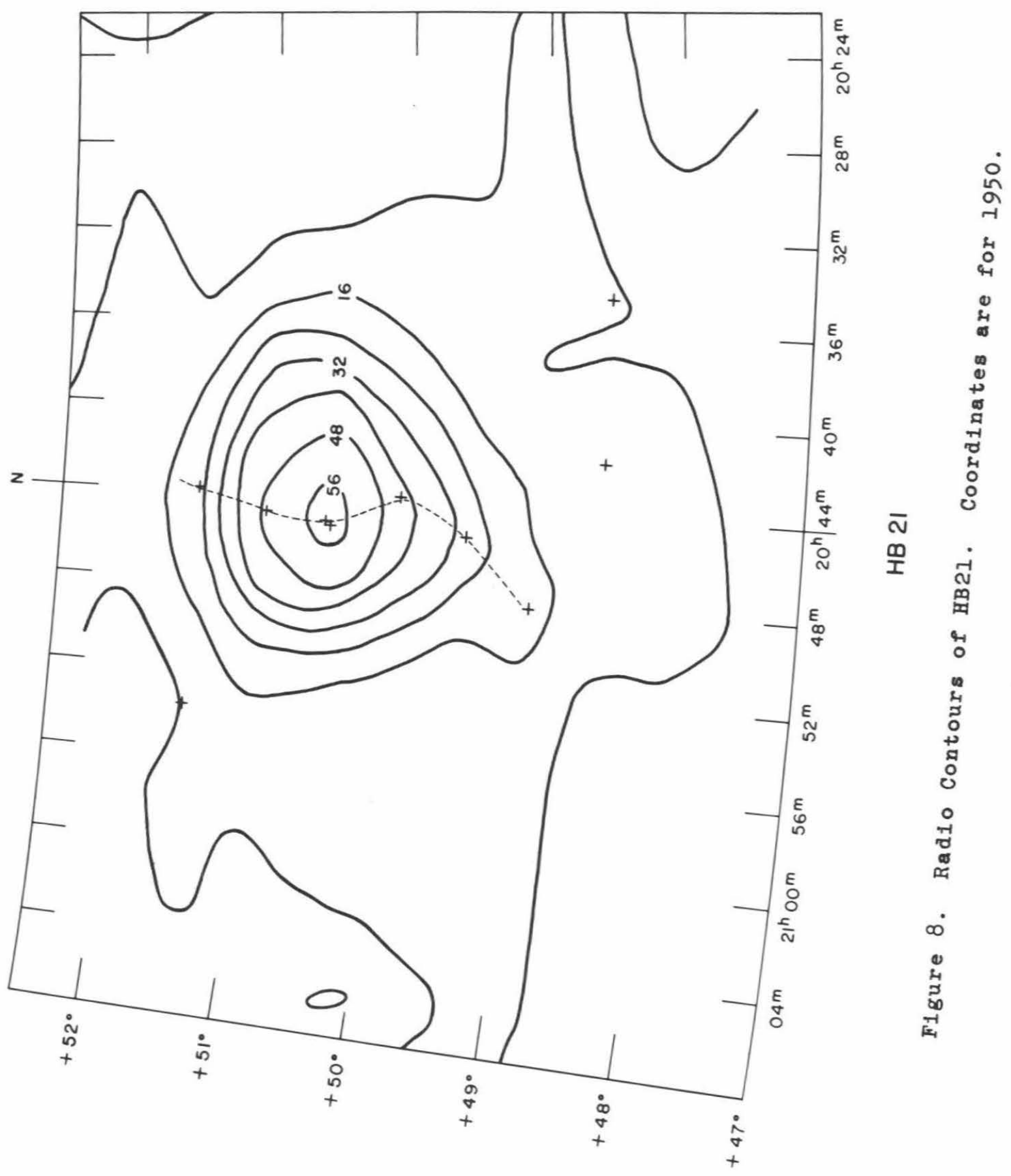




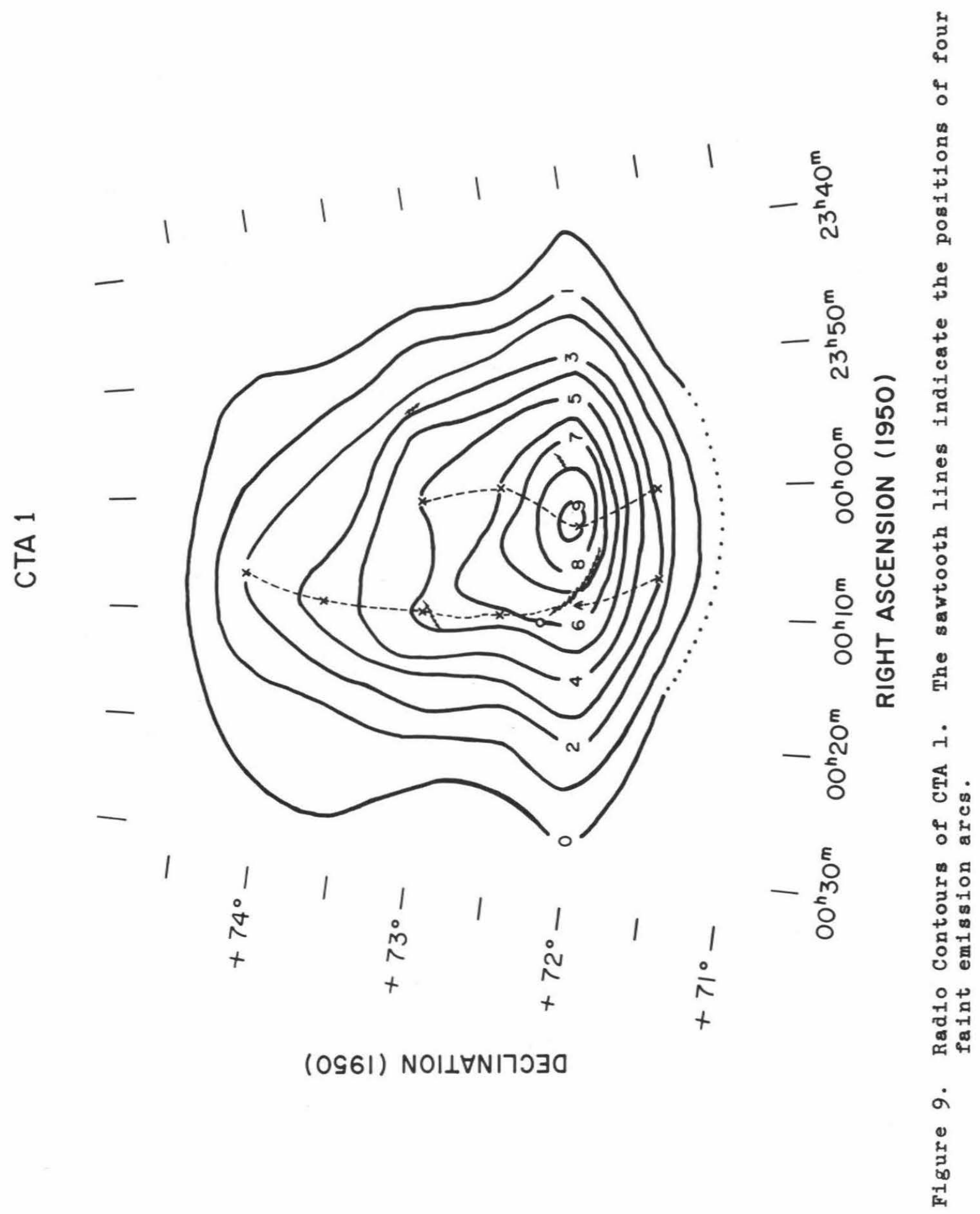




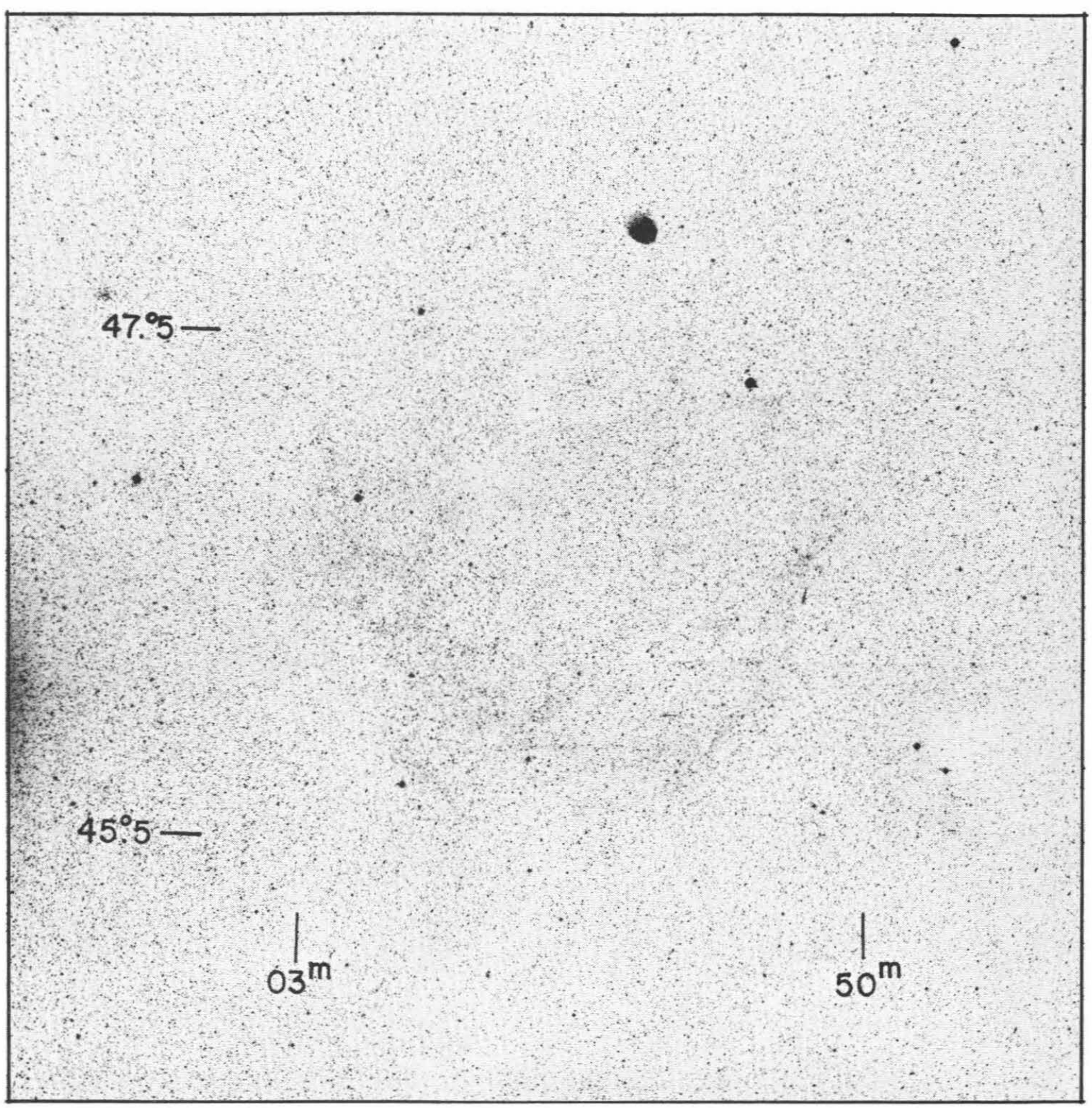

Figure 10. Photograph of HB9. Reproduced from a 48" deep red plate. North is at the top, east at the left. The coordinate marks (1950) indicate the approximate extent of the source. 


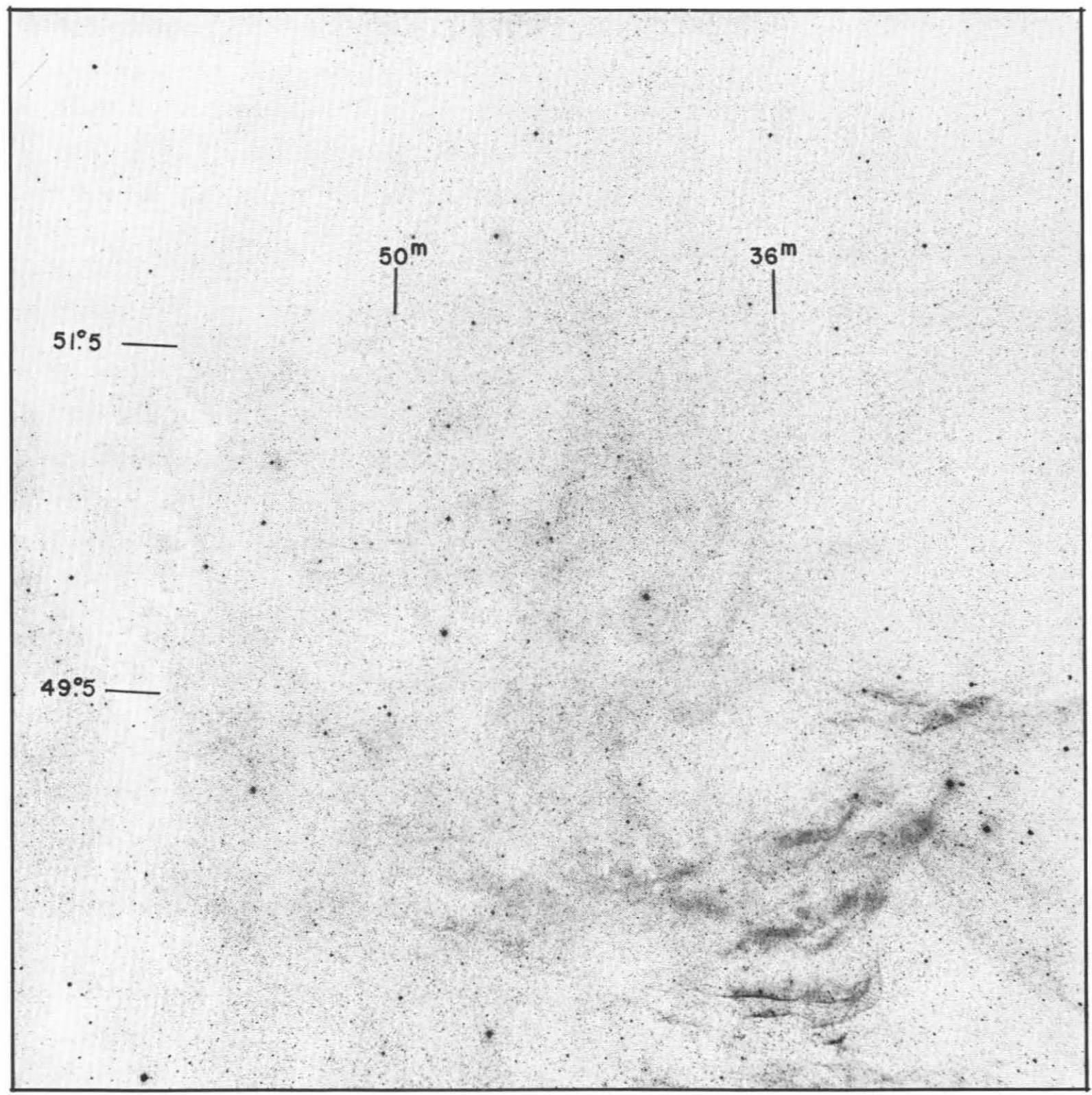

Figure 11. Photograph of HB2l. The prominent emission in the lower right (SW) is not associated with the radio source. 
Abell, G. 1960, private communication.

Baade, W. 1956 , B.A.N., XII, 312.

Baade, W. and Minkowski, R. 1954, Ap.J., $119,206$.

Baldwin, J. E. 1955, M.N., 115, 690.

Baldwin, J. E., and Leslie, P. R. R. 1960, M.N., $120,72$.

Biraud, F., Lequeux, J., and LeRoux, E. 1960, Obs., $80,116$.

Bok, B. J., and Warwick, C. 1957, A.J., 62, 323.

Bolton, J. G. 1960, private communication.

Bolton, J. G., and Stanley, G. J. 1949, Aust. Journal of Sci. Res., 2. 139.

Brown, R. Hanbury, Davies, R. D., and Hazard, C. 1960, Observatory, $80,191$.

Brown, R. Hanbury, and Hazard, C. 1953, M.N., $113,123$.

Chamberlain, J. W. 1953a, Ap.J., $\frac{117}{\mathrm{~mm}}, 387$.

Chamberlain, J. W. 1953b, Ap.J., 117 , 399.

Eaton, J. J., and Kraus, J. D. 1959, Ap.J., 129, 284.

Edge, D. O., Shakeshaft, J. R., McAdam, W. B., Baldwin, J. E., and Archer, S. 1959, Mem. R.A.S., 68, 37.

Hagen, J. P., McClain, E. F., and Hepburn, N. 1954, Proc.I.R.E., 42,1811 .

Harris, D. E. 1961, Thesis submitted to the California Institute of Technology.

Harris, D. E., and Roberts, J. A. 1960, Pub.A.S.P., 72, 237.

Leslie, P. R. R. 1960, Observatory, $80,23$.

Mathewson, D. S., Large, M. I., and Haslam, C. G. T. 1961,

M.N., 121,543 .

Mills, B. Y., and Slee, O. B. 1957, A.J.P., 10, 162.

Mills, B. Y., Slee, O. B., and Hill, E. R. 1960, A.J.P., 13, 676.

Minkowski, R. 1958, Rev.Mod.Phy., 30, 1048.

Minkowski, R. 1959, Paris Symposium on Radio Astronomy, p. 315 .

Opik, E. J. 1953, I.A.J., 2, 219.

Osterbrock, D. E. 1958, Pub.A.S.P., 70, 180.

Osterbrock, D. E., and Stockhausen, R. E. 1960, Ap.J., 131, 310.

Piddington, J. H., and Trent, G. H. 1956, A.J.P., 9, 74.

Rishbeth, H. 1956, A.J.P., 9, 494. 
Rishbeth, H. 1958, A.J.P., 11 1750.

Seeger, C. L., Westerhout, G., and van de Hulst, H. C. 1956 , B.A.N., 13,89 .

Sheridan, K. V. 1958, A.J.P., 11 , 400.

Shklovsky, I. S. 1960a, Soviet Astronomy, $4,243$.

(Russ.) 1960, Astr.Zhurnal, 37, 256.

Shklovsky, I. S. 1960b, Soviet Astronomy, $4,355$.

(Russ.) 1960, Astr.Zhurnal, 37, 369.

Van den Bergh, S. 1960, ze1t.fll Astrophysik, $511,15$.

Walsh, D., and Brown, R. Hanbury 1955, Nature, $175,808$.

Westerhout, G. 1958, B.A.N., $144,215$.

Westerhout, G. 1960, private communication.

Whitfield, G. R. 1957, M.N., $1177,680$.

Wilson, R. W., and Bolton, J. G. 1960, Pub.A.S.P., 72, 331.

Woltjer, I. 1958, B.A.N., $\frac{1}{\mathrm{~m}} 4$, No. $483,39$. 